\title{
Emergence and Phylogenetic Analysis of Amantadine-Resistant Influenza A Subtype H3N2 Viruses in Dublin, Ireland, over Six Seasons from 2003/2004 to 2008/2009
}

\author{
A. Salter ${ }^{a, b} \quad$ B. Ni Laoib $\quad$ B. Crowley ${ }^{\text {b, c }}$ \\ a Department of Clinical Microbiology, Trinity College Dublin, and ' ${ }^{\text {b }}$ epartment of Microbiology, \\ Central Pathology Laboratory, St. James's Hospital, and 'National Virus Reference Laboratory, \\ University College Dublin, Dublin, Ireland
}

\section{Key Words \\ Amantadine resistance $\cdot$ Irish influenza A viruses $\cdot$ Matrix 2 gene $\cdot$ Haemagglutinin gene $\cdot$ Phylogenetic analysis • Clades $\mathrm{S}$ and $\mathrm{N} \cdot$ Antigenic variations}

\begin{abstract}
Objectives: To determine the prevalence of amantadine-resistant influenza $A$ viruses and perform genetic analysis of isolates collected in Dublin during six seasons (2003/2004 to 2008/2009). Methods: Known mutations in the matrix 2 gene (M2) conferring amantadine resistance were screened and phylogenetic analysis of the haemagglutinin gene ( $\mathrm{HA})$ performed. Results: Of 1,180 samples, 67 influenza A viruses were isolated, $88 \%$ of which were subtype H3N2. Amantadine resistance was only found in subtype H3N2 and increased dramatically from $7 \%$ in $2003 / 2004$ to $90 \%$ in $2008 / 2009$. A maximum likelihood tree of the HA gene of influenza A H3N2 isolates differentiated them into two distinct clades, clade $\mathrm{N}$ and clade $\mathrm{S}$, where the majority of isolates were amantadine-resistant and amantadine-sensitive, respectively. The clades were distinguished by amino acid substitutions, S193F and D225N, which probably conferred a selective advantage for the spread of such viruses. Phyloge-
\end{abstract}

netic analysis showed some degree of antigenic drift when compared with the vaccine strain of the corresponding season. Conclusions: This study showed that circulation in Ireland of a distinct lineage, clade $\mathrm{N}$, among $\mathrm{H} 3 \mathrm{~N} 2$ viruses favoured emergence of amantadine resistance. Furthermore, comparison of circulating Irish viruses and vaccine strains used in the northern hemisphere showed high similarity.

Copyright $\odot 2011$ S. Karger AG, Basel

\section{Introduction}

Two classes of antiviral drugs are licensed for prophylaxis and treatment of influenza virus infections in the Republic of Ireland. The matrix 2 (M2) ion channel inhibitors (MI) include adamantane derivatives, amantadine and rimantadine, and have been used for more than 30 years [1]. The other class of antiviral agents, the neuraminidase inhibitors (NI), includes zanamivir and oseltamivir which were licensed for use a decade ago [2]. Although adamantane antivirals are effective in treating the symptoms of influenza virus if administered within $24 \mathrm{~h}$ of onset of illness, the potential for rapid development of resistance in this class of drugs restricts its value

\section{KARGER}

Fax +4161306 1234

E-Mail karger@karger.ch

www.karger.com (c) 2011 S. Karger AG, Basel

0300-5526/11/0546-0305\$38.00/0

Accessible online at:

www.karger.com/int
B. Crowley

Department of Microbiology

Central Pathology Laboratory

St. James's Hospital, Dublin 8 (Ireland)

Tel. +353 14162 966, E-Mail bcrowley@stjames.ie 
in treatment [3]. However, in the 2005-2006 influenza season, a high incidence of amantadine resistance was noted in the United States, where $92 \%$ of 209 influenza A $(\mathrm{H} 3 \mathrm{~N} 2)$ isolates had molecular evidence of resistance to amantadine [4]. This antiviral resistance appeared without prior pressure from widespread use of adamantanes and disseminated rapidly in the population. Similarly in Japan, during the same season, a high prevalence (65.3\%) and wide circulation of amantadine-resistant influenza A $\mathrm{H} 3 \mathrm{~N} 2$ virus were recorded [5]. In addition, the amantadine-resistant strains in Japan belonged to a phylogenetic group named clade $\mathrm{N}$, and were characterized by amino acid substitutions in haemagglutinin (HA) at residues 193 and 225 where serine replaced phenylalanine and aspartic acid replaced asparagine, respectively [5]. Furthermore, a high percentage of influenza A H3N2 viruses circulating in Eastern Europe have shown resistance to adamantanes in recent years [6]. Therefore, this high incidence of adamantane resistance globally led to a recommendation that amantadine and rimantadine no longer should be used for prophylaxis and treatment of influenza A infections [4]. To date, there are no data on molecular analysis of both subtypes of seasonal influenza A in Dublin, Ireland. This study is a laboratory-based analysis of influenza A viruses conducted to clarify the seasonal influenza patterns and prevalence of amantadineresistant influenza A viruses over six winter seasons. Furthermore, a longitudinal study of genetic changes in the HA molecule of amantadine-sensitive and amantadineresistant strains was also performed.

\section{Materials and Methods}

A total of 1,180 samples, including nasophayngeal aspirates, combined nose/throat swabs and bronchoalveolar lavages, were collected for laboratory diagnosis of respiratory viruses at St. James's Hospital, Dublin between November 2003 and March 2009. This is the largest tertiary-care centre in the Republic of Ireland to which patients from all areas of Ireland are referred. Furthermore, the study included samples from patients living in different areas of Dublin city and surrounding counties (demographic data not shown). Eighty-seven samples were positive for influenza A, while no sample was positive for influenza B virus. These samples were confirmed as positive for influenza A virus by direct immunofluorescence (Imagen ${ }^{\mathrm{TM}}$ Influenza virus A and $B$, Oxoid). In addition, the samples were inoculated into MadinDarby canine kidney (MDCK) cells. A positive cytopathic effect was confirmed as influenza virus positive by staining MDCK cells using monoclonal antibodies to influenza A and B (Imagen Influenza virus A and B, Oxoid). Altogether, 67 samples collected from 64 patients were available for analysis. A 371-bp portion of the M2 gene from these 67 influenza A-positive isolates was am- plified by PCR. Phylogenetic analysis of a maximum likelihood tree constructed from the amplified partial M2 gene product (371 bp) allowed subtyping of the 67 influenza A-positive isolates (fig. 1). The latter confirmed that 59 influenza A viruses belonged to subtype $\mathrm{H} 3 \mathrm{~N} 2$ while the remaining 8 were subtype H1N1. Oligonucleotide primers used for amplification of M2 and HA sequences of influenza A subtype H3N2 were designed using Primer 3 software (http://frodo.wi.mit.edu) (table 1). The forward primer MF1 and reverse primer MR1027 generated a 1,027-bp amplicon. A second forward primer, MF 656, was designed for use in a heminested PCR on samples that failed to amplify product in the first round of PCR. This round 2 primer pair (MF656 + MR1027) gave a product size of $371 \mathrm{bp}$. The round 1 and 2 amplicon sizes of the HA gene of H3N2 isolates were 989 and 737 bp, respectively. Amplification was carried out on cDNA extracts using the HotStar HiFidelity Polymerase Kit (Qiagen, Hilden, Germany). All PCR reactions were run on either the GeneAmp 9700 PCR System thermal cycler or the Veriti Thermal Cycler (Applied Biosystems, Foster City, Calif., USA). A preextracted nontemplate control was included with each run. Both first and second round cycling parameters for each gene were identical and consisted of an initial temperature of $95^{\circ}$ for $5 \mathrm{~min}$, followed by 35 cycles of $94^{\circ}$ for $15 \mathrm{~s}$ (denaturation), $55^{\circ}$ for $1 \mathrm{~min}$ (primer annealing) and $72^{\circ}$ for $1 \mathrm{~min}$ (extension) and a final elongation step of $72^{\circ}$ for $10 \mathrm{~min}$.

Phylogenetic reconstructions, all employing maximum likelihood, were carried out using PAUP*4.0b10 [7]. Multiple sequence alignments were performed using the alignment function in the Lasergene ${ }^{\circledR}$ MegAlign software (DNASTAR, Madison, Wis., USA). The 'best fit' models of evolution for the aligned datasets were determined using the Modeltest version 3.06 [8] by comparing the likelihood of the different parameters for all models of substitution. Model parameters were then estimated using a heuristic search strategy and selecting those with the highest likelihood. Bootstrap resampling was carried out using PAUP* with 1,000 replicates. Phylogenetic analysis of the M2 gene (371 bp) was carried out on all the 67 influenza $A$ isolates employing a swinelineage influenza A strain, A/Aragon/RR3218/2008 (GenBank FJ713784) as the outgroup. The model of evolution chosen by hLRT using Modeltest was K80+G. Phylogenetic analysis of the HA gene (989 bp) was carried out on 13 of the 59 influenza A H3N2 isolates employing the vaccine strain A/Panama/2007/1999 (GenBank EF626612) as the outgroup. The model of evolution chosen by hLRT using Modeltest was TVM+G.

Fig. 1. A maximum likelihood tree based on the partial M2 gene ( $371 \mathrm{bp)}$ of 67 influenza A isolates collected from St. James's Hospital between the 2003/2004 and 2008/2009 influenza seasons using A/Aragon/RR3218/2008 (GenBank FJ713784) as an outgroup. Bootstrap resampling was carried out using 1,000 replicates of the dataset and the values are written as a percentile beside the appropriate branch. Bootstrap values $<70 \%$ are not shown. Several representative influenza $\mathrm{A} \mathrm{H} 1 \mathrm{~N} 1$ and $\mathrm{H} 3 \mathrm{~N} 2$ isolates were included. 0 Adamantane-resistant $(\mathrm{S} 31 \mathrm{~N})$ isolates; $\mathbf{0}=$ isolates with the S23N, V28I and K56S amino acid substitutions. 


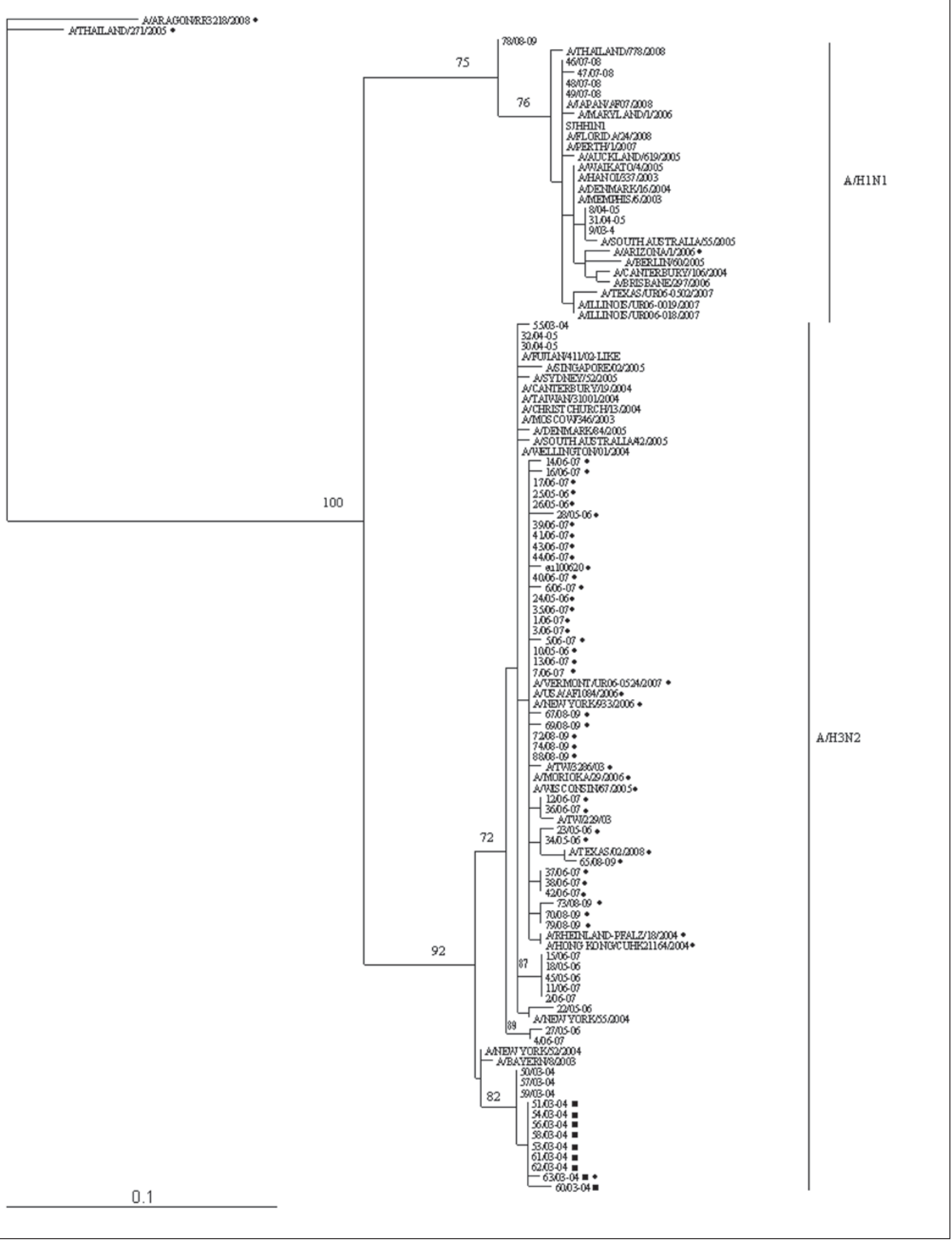

Genetic Analysis of

Intervirology 2011;54:305-315

Amantadine-Resistant Influenza A 
Table 1. Primers used in the amplification of different regions of all influenza viruses in this study

\begin{tabular}{llllc}
\hline Primer name & Target gene & Primer sequence 5' -3' $^{\prime}$ & Primer function & Nucleotide position \\
\hline MF1 & matrix gene (M2) & AGCAAAAGCAGGTAGATATTG & sense & $1-21$ \\
MF 656 & & TTGGGACTCATCCTAGCTCCAG & sense & $656-678$ \\
MR 1027 & & AGTAGAAACAAGGTAGTTTTT & antisense & $1027-1005$ \\
\hline H3 78 F1 & HA & GAAAACAATCACGAATGACCAA & sense & $78-100$ \\
H3 222 F2 & gene for & TCAGTGTGATGGCTTCCAAA & sense & $222-242$ \\
H3 345 F SEQ & H3N2 subtypes & CGGCACACTGGAGTTTAACA & sequencing & $345-365$ \\
H3 720 R SEQ & & TCCCGGTTTTACTATTGTCCA & sequencing & $720-700$ \\
H3 1067 R1 & & TGATGCCTGAAACCATACCA & antisense & $1067-1047$ \\
H3 959 R2 & & ATCCCTGTTGCCAATTTCAG & antisense round 2 & $959-939$ \\
\hline
\end{tabular}

\section{Results}

\section{Molecular Screening for Amantadine-Resistant Influenza A Viruses}

Of the 1,180 samples screened for respiratory viruses between 2003/2004 and 2008/2009, 87 were influenza Apositive, of which 67 were available for analysis. A total of $38(57 \%)$ had the most commonly reported amantadine resistance mutation, $\mathrm{S} 31 \mathrm{~N}$ (tables 2, 3). All of the resistant strains belonged to the H3N2 subtype. Between 2003/2004 and $2008 / 2009$, there was a trend towards an increase in levels of resistance to amantadine, beginning in 2005/ 2006 (table 2). In the 2003/2004 season, only one isolate $(1 / 14 ; 7 \%)$ had molecular evidence of resistance (table 2). No resistant isolates were detected in 2004/2005; however, 73\% (8/11) of influenza A-positive samples had the S31N mutation in $2005 / 2006$. Similarly, in $2006 / 2007$, there was clear evidence of the predominance of amantadine-resistant isolates with $83 \%(20 / 24)$ of influenza Apositive samples carrying the $\mathrm{S} 31 \mathrm{~N}$ mutation in the M2 protein. While no adamantane resistance was detected in the $2007 / 2008$ season, only 4 samples positive for influenza A virus were identified, leaving analysis of the season incomplete. However, during the 2008/2009 season, there was a continued trend to high levels of resistance to amantadine (90\%), with genotypic evidence of resistance in the M2 gene at codon 31. While the well-recognized resistance mutation, $\mathrm{S} 31 \mathrm{~N}$, was found in all resistant strains, 1 sample, 14/06-07, had dual resistance mutations, sometimes called a double resistance mutation, with V27A and S31N in the M2 (tables 3, 4).

Another amino acid substitution of particular interest occurred at amino acid 51 (I51V) (table 4). It was reported previously in influenza A H3N2 in Hong Kong in 2000 and was detected with increasing frequency between
Table 2. Summarized results of amantadine-resistant influenza A H3N2 viruses in St. James's Hospital Dublin (2003-2009)

\begin{tabular}{lllll}
\hline Flu season & $\begin{array}{l}\text { Total } \\
\text { samples, } \mathrm{n}\end{array}$ & $\begin{array}{l}\text { Resistant } \\
\text { samples, } \mathrm{n}\end{array}$ & $\begin{array}{l}\text { Sensitive } \\
\text { samples, } \mathrm{n}\end{array}$ & $\begin{array}{l}\text { Resis- } \\
\text { tance, \% }\end{array}$ \\
\hline $2003 / 2004$ & 14 & 1 & 13 & 7 \\
$2004 / 2005$ & 4 & 0 & 4 & 0 \\
$2005 / 2006$ & 11 & 8 & 3 & 73 \\
$2006 / 2007$ & 24 & 20 & 4 & 83 \\
$2007 / 2008$ & 0 & 0 & 4 & 0 \\
$2008 / 2009$ & 10 & 9 & 1 & 90 \\
\hline Total & 67 & 38 & 29 & 57 \\
\hline
\end{tabular}

2000 and 2006 [9]. In the current study, it was found to coexist with the resistance mutation $\mathrm{S} 31 \mathrm{~N}$ in most samples. However, 1 resistant sample from the 2003/2004 (63/03-04) with acquired resistance at S31N did not have the I51V substitution. I51V did not occur in combination with any of the other known amantadine-resistant mutations (L26F, V27A, A30T, G34E) or polymorphisms in the M2 gene (S23N, V28I, K56R).

The vast majority of amantadine-resistant influenza A $\mathrm{H} 3 \mathrm{~N} 2$ isolates had not received prior treatment with an adamantane antiviral agent. The latter reflected inherent resistance to amantadine among influenza A H3N2 circulating worldwide. However, 1 patient developed resistance to amantadine in the 2003/2004 season at 10 weeks' postallogenic haematopoietic stem cell transplant. As part of the conditioning regime he received alemtuzumab, humanized monoclonal antibody to CD52, which induced prolonged lymphopenia (absolute lymphocyte count $<0.3 \mathrm{~mm}^{3}$ ). 
Table 3. Frequency of amantadine resistance mutations in $M$ gene in samples collected at St. James's Hospital Dublin (2003-2009)

\begin{tabular}{lllllll}
\hline \multirow{2}{*}{$\begin{array}{l}\text { M2/amantadine- } \\
\text { resistant mutation }\end{array}$} & \multicolumn{5}{l}{ Year (number of isolates examined) } \\
\cline { 2 - 6 } & $2003 / 2004(13)$ & $2004 / 2005(4)$ & $2005 / 2006(11)$ & $2006 / 2007(24)$ & $2007 / 2008(4)$ & $2008 / 2009(8)$ \\
\hline L26F & 0 & 0 & 0 & 0 & 0 & 0 \\
V27A & 0 & 0 & 0 & $1(4 \%)^{\mathrm{a}}$ & 0 & 0 \\
A30T & 0 & 0 & 0 & 0 & 0 & 0 \\
S31N & $1(7 \%)$ & 0 & $8(73 \%)$ & $20(83 \%)$ & 0 & $9(90 \%)$ \\
G34E & 0 & 0 & 0 & 0 & 0 & 0 \\
\hline
\end{tabular}

a Sample in 2006/2007 had a double V27A/S31N resistance mutation.

Phylogenetic Analysis of the HA1 Portion of the HA Gene of Influenza A H3N2 Isolates

In this study, 14 isolates were selected for sequencing of the HA1 subunit of the HA gene between amino acids 11 and 295. These sequences included both amantadinesensitive and amantadine-resistant strains. Amino acid sequences were compared to vaccine strain A/Pana$\mathrm{ma} / 2007 / 1999$ (table 5) and phylogenetic trees were constructed (fig. 2). No samples were included from the 2007/2008 season as all positive samples were subtype H1N1. Phylogenetic tree analysis of the HA gene of influenza A H3N2 viruses showed two different lineages, termed 'clade N' [10] and 'clade S' [5] (fig. 2). The amino acid changes that defined clade $\mathrm{N}$ viruses were serine (AGC) to phenylalanine (TTC) at position 193 (S193F) and aspartic acid (GAT) to asparagine (AAT) at position 225 (D225N) (table 5). In this study, the majority of amantadine-resistant viruses contained the $\mathrm{S} 31 \mathrm{~N}$ mutation and clustered into clade $\mathrm{N}(10 / 05-06,1 / 06-07,14 / 06-07$, 72/08-09, 73/08-09 and 65/08-09). However, one virus from the 2006/2007 season, with no S31N mutation in the M2 protein, 2/06-07, also had both the S193F and D225N amino acid substitutions that grouped it into clade $\mathrm{N}$ (fig. 2). This was an interesting finding since the majority of clade $\mathrm{N}$ strains are known to be amantadine resistant [5].

Within clades $\mathrm{N}$ and $\mathrm{S}$, samples also clustered into separate groups depending on the influenza season during which they were isolated and on amino acid changes that defined these groups (fig. 2; table 5). All partial HA1 amino acid sequences of the 14 influenza A H3N2 isolates were compared to vaccine strain A/Panama/2007/ 1999 to determine key substitutions that may be responsible for their separation into different groups within the phylogenetic tree (fig. 2). There were 11 positions in the
HA1 protein sequence of all the isolates examined where amino acid changes were detected when compared with A/Panama/2007/1999 (table 5). These were at positions $50,75,83,131,155,156,183,186,202,222$ and 225. The antigenic sites A-E on the HA1 of influenza A H3N2 viruses which confer antigenic variability have been described previously $[11,12]$. The clade S sensitive 2003/ 2004 isolates (54/03-04 and 50/03-04) and the resistant 2003/2004 isolate (63/03-04) had a high degree of homology with vaccine strain A/Fujian/411/2002 but had a significant amino acid substitution compared to the A/Panama/2007/1999 vaccine strain at position 126 of antigenic site A (table 5). The majority of samples examined after the 2003/2004 season had amino acid substitutions at positions 145, 159, 189, 226 and 227 when compared with the A/Fujian/411/2002-like samples of 2003/2004. Amino acid position 145 was in antigenic site $A, 159$ and 189 were located at antigenic site B, while positions 226 and 227 were at antigenic site D [10-12]. The clade S 2004/2005 sample, 30/04-05, closely resembled the vaccine strain of that season, A/California/7/2004. The other sensitive isolates in clade S, 2004/2005 (32/04-05) and 2005/2006 (27/05-06), had amino acid substitutions at positions 112 and 173 when compared to vaccine strain A/Pana$\mathrm{ma} / 2007 / 1999$. These samples, 30/04-05, 32/04-05 and $27 / 05-06$, were also genetically close to the vaccine strain A/Wellington/1/2004 but differed antigenically from this reference strain. This finding is in accordance with the substitution, by the WHO, of A/Wellington/1/2004 for A/California/7/2004 in the 2005/2006 season influenza vaccine composition [14].

The 2005/2006 resistant strain (10/05-06) within clade $\mathrm{N}$ had a high degree of similarity with the $2005 \mathrm{vac}-$ cine strain A/Wisconsin/67/2005. Amino acid changes at positions 50 and 140 were seen in all resistant samples 
Table 4. Amino acid variations in M2 of amantadine-resistant influenza A H3N2 viruses in this study

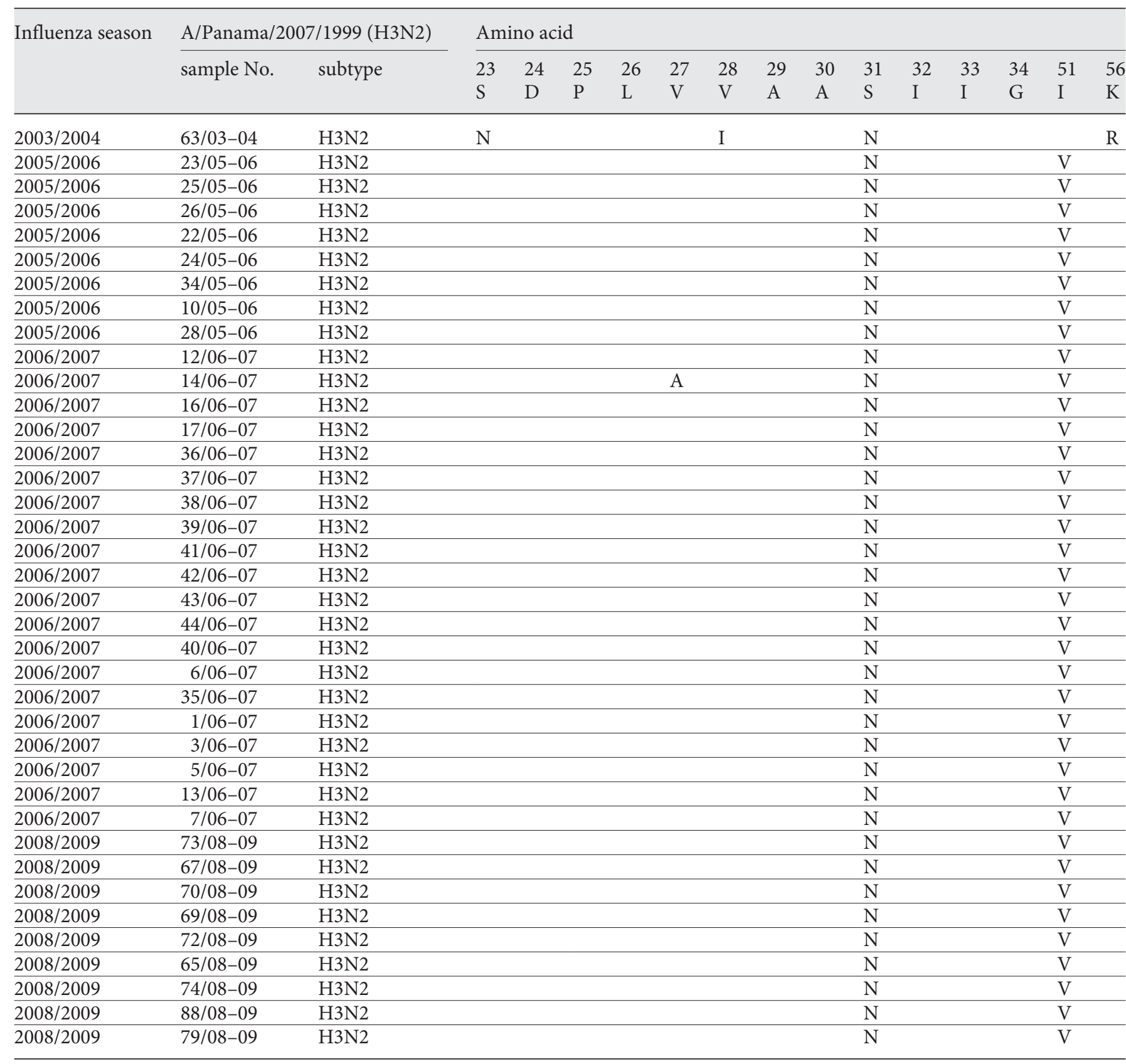

in $2006 / 2007(1 / 06-07$ and $14 / 06-07)$ and 2008/2009 (72/08-09, 73/08-09 and 65/08-09) when compared with 2005/2006 A/Wisconsin/67/2005-like samples. These samples (1/06-07, 14/06-07, 72/08-09, 73/08-09 and 65/08-09) clustered together with the 2008-2009 northern hemisphere season vaccine strain A/Brisbane/10/ 2007. Interestingly, the amantadine-sensitive sample that clustered into clade N, 2/06-07, had three further amino acid substitutions at positions 142,157 and 173 . This isolate was determined to be in clade $\mathrm{N}$ based on the presence of the S193F and D225N substitutions in the HA1 region of the HA gene. 
Table 5. Amino acid variations in the HA1 subunit of HA protein of $14 \mathrm{H} 3 \mathrm{~N} 2$ influenza A-positive samples collected between 2003 and 2009 winter seasons compared to the vaccine strain of the corresponding season

\begin{tabular}{|c|c|c|c|c|c|c|c|c|c|c|c|c|c|c|c|c|c|c|c|c|c|c|c|c|c|c|c|c|c|c|}
\hline \multirow[t]{3}{*}{ Virus } & \multirow[t]{3}{*}{ S31N } & \multicolumn{29}{|c|}{ Antigenic sites } \\
\hline & & $\mathrm{C}$ & & $\mathrm{E}$ & $\mathrm{E}$ & & A & A & A & A & A & B & B & B & B & $\mathrm{D}$ & $\mathrm{D}$ & & B & B & B & & & & & & $\mathrm{D}$ & $\mathrm{D}$ & $\mathrm{E}$ & \\
\hline & & 50 & 62 & 75 & 83 & 112 & 126 & 131 & 140 & 142 & 145 & 155 & 156 & 157 & 159 & 173 & 174 & 183 & 186 & 188 & 189 & 193 & 199 & 202 & 222 & 225 & 226 & 227 & 261 & 282 \\
\hline A/Panama/2007/1999 & & $\mathrm{R}$ & $\mathrm{E}$ & $\mathrm{H}$ & $\mathrm{E}$ & $\mathrm{V}$ & $\mathrm{N}$ & A & K & $\mathrm{R}$ & K & $\mathrm{H}$ & Q & $\mathrm{L}$ & $\mathrm{Y}$ & $\mathrm{K}$ & $\mathrm{F}$ & $\mathrm{L}$ & S & $\mathrm{D}$ & S & S & S & $\mathrm{V}$ & $\mathrm{W}$ & G & $\mathrm{V}$ & S & $\mathrm{R}$ & $\mathrm{T}$ \\
\hline A/Fujian/411/2002 & & G & $\mathrm{E}$ & Q & $\mathrm{K}$ & $\mathrm{V}$ & $\mathrm{N}$ & $\mathrm{T}$ & $\mathrm{K}$ & $\mathrm{R}$ & $\mathrm{K}$ & $\mathrm{T}$ & $\mathrm{H}$ & $\mathrm{L}$ & $\mathrm{Y}$ & $\mathrm{K}$ & $\mathrm{F}$ & $\mathrm{H}$ & G & $\mathrm{D}$ & S & S & $S$ & I & $\mathrm{R}$ & $\mathrm{D}$ & $\mathrm{V}$ & $S$ & $\mathrm{R}$ & $\mathrm{T}$ \\
\hline A/California/7/2004 & & G & $\mathrm{E}$ & Q & $\mathrm{K}$ & $\mathrm{V}$ & $\mathrm{N}$ & $\mathrm{T}$ & $\mathrm{K}$ & $\mathrm{R}$ & $\mathrm{N}$ & $\mathrm{T}$ & $\mathrm{H}$ & $\mathrm{L}$ & $\mathrm{F}$ & $\mathrm{K}$ & $\mathrm{F}$ & $\mathrm{H}$ & G & $\mathrm{N}$ & $\mathrm{N}$ & S & $\mathrm{N}$ & $\mathrm{I}$ & $\mathrm{R}$ & $\mathrm{D}$ & $\mathrm{I}$ & $\mathrm{P}$ & $\mathrm{R}$ & $\mathrm{T}$ \\
\hline $\mathrm{A} / \mathrm{Wisconsin} / 67 / 2005$ & & G & $\mathrm{E}$ & $\mathrm{Q}$ & $\mathrm{K}$ & $\mathrm{V}$ & $\mathrm{N}$ & $\mathrm{T}$ & $\mathrm{K}$ & $\mathrm{R}$ & $\mathrm{N}$ & $\mathrm{T}$ & $\mathrm{H}$ & $\mathrm{L}$ & $\mathrm{F}$ & $\mathrm{K}$ & $\mathrm{F}$ & $\mathrm{H}$ & G & $\mathrm{D}$ & $\mathrm{N}$ & $\mathrm{F}$ & $\mathrm{N}$ & $\mathrm{I}$ & $\mathrm{R}$ & $\mathrm{N}$ & I & $\mathrm{P}$ & $\mathrm{R}$ & $\mathrm{T}$ \\
\hline A/Brisbane/10/2007 & & $\mathrm{E}$ & $\mathrm{E}$ & Q & $\mathrm{K}$ & $\mathrm{V}$ & $\mathrm{N}$ & $\mathrm{T}$ & I & $\mathrm{R}$ & $\mathrm{N}$ & $\mathrm{T}$ & $\mathrm{H}$ & $\mathrm{L}$ & $\mathrm{F}$ & $\mathrm{K}$ & $\mathrm{F}$ & $\mathrm{H}$ & G & $\mathrm{D}$ & $\mathrm{N}$ & $\mathrm{F}$ & $\mathrm{N}$ & I & $\mathrm{R}$ & $\mathrm{N}$ & I & $\mathrm{P}$ & $\mathrm{R}$ & $\mathrm{T}$ \\
\hline 50/03-04 & & G & G & Q & $\mathrm{K}$ & & $\mathrm{D}$ & $\mathrm{T}$ & & & & $\mathrm{T}$ & $\mathrm{H}$ & & & & & $\mathrm{H}$ & G & & & & & I & $\mathrm{R}$ & $\mathrm{D}$ & & & & \\
\hline 54/03-04 & & G & & Q & $\mathrm{K}$ & & $\mathrm{D}$ & $\mathrm{T}$ & & & & $\mathrm{T}$ & $\mathrm{H}$ & & & & & $\mathrm{H}$ & G & & & & & I & $\mathrm{R}$ & $\mathrm{D}$ & & & & \\
\hline 55/03-04 & & G & & Q & $\mathrm{K}$ & & & $\mathrm{T}$ & & & & $\mathrm{T}$ & $\mathrm{H}$ & & $\mathrm{F}$ & & & $\mathrm{H}$ & G & & $\mathrm{N}$ & & & I & $\mathrm{R}$ & $\mathrm{D}$ & & $\mathrm{P}$ & & \\
\hline 63/03-04 & $\mathrm{X}$ & G & & Q & $\mathrm{K}$ & & $\mathrm{D}$ & $\mathrm{T}$ & & & & $\mathrm{T}$ & $\mathrm{H}$ & & & & & $\mathrm{H}$ & G & $\mathrm{Y}$ & & & & I & $\mathrm{R}$ & $\mathrm{D}$ & & & & \\
\hline 30/04-05 & & $\mathrm{G}$ & & Q & $\mathrm{K}$ & & & $\mathrm{T}$ & & & $\mathrm{N}$ & $\mathrm{T}$ & $\mathrm{H}$ & & $\mathrm{F}$ & & $\mathrm{Y}$ & $\mathrm{H}$ & G & & $\mathrm{N}$ & & & I & $\mathrm{R}$ & $\mathrm{D}$ & I & $\mathrm{P}$ & & \\
\hline 32/04-05 & & G & & Q & $\mathrm{K}$ & I & & $\mathrm{T}$ & & & $S$ & $\mathrm{~T}$ & $\mathrm{H}$ & & $\mathrm{F}$ & E & & $\mathrm{H}$ & G & & $\mathrm{N}$ & & & $\mathrm{I}$ & $\mathrm{R}$ & $\mathrm{D}$ & $\mathrm{I}$ & $\mathrm{P}$ & & \\
\hline 10/05-06 & $\mathrm{X}$ & G & & Q & $\mathrm{K}$ & & & $\mathrm{T}$ & & & $\mathrm{N}$ & $\mathrm{T}$ & $\mathrm{H}$ & & $\mathrm{F}$ & & & $\mathrm{H}$ & G & & $\mathrm{N}$ & $\mathrm{F}$ & & I & $\mathrm{R}$ & $\mathrm{N}$ & $\mathrm{I}$ & $\mathrm{P}$ & & \\
\hline 27/05-06 & & G & $\mathrm{K}$ & Q & $\mathrm{K}$ & I & & $\mathrm{T}$ & & & $\mathrm{N}$ & $\mathrm{T}$ & $\mathrm{H}$ & & $\mathrm{F}$ & E & & $\mathrm{H}$ & G & & $\mathrm{N}$ & & $\mathrm{P}$ & $\mathrm{I}$ & $\mathrm{R}$ & $\mathrm{D}$ & $\mathrm{I}$ & $\mathrm{P}$ & & \\
\hline $1 / 06-07$ & $\mathrm{X}$ & E & & Q & $\mathrm{K}$ & & & $\mathrm{T}$ & I & & $\mathrm{N}$ & $\mathrm{T}$ & $\mathrm{H}$ & & $\mathrm{F}$ & & & $\mathrm{H}$ & G & & $\mathrm{N}$ & $\mathrm{F}$ & & $\mathrm{I}$ & $\mathrm{R}$ & $\mathrm{N}$ & $\mathrm{I}$ & $\mathrm{P}$ & & \\
\hline $14 / 06-07$ & $\mathrm{X}$ & E & & Q & $\mathrm{K}$ & & & $\mathrm{T}$ & I & & & $\mathrm{T}$ & $\mathrm{H}$ & & $\mathrm{F}$ & & & $\mathrm{H}$ & G & & & $\mathrm{F}$ & & $\mathrm{I}$ & $\mathrm{R}$ & $\mathrm{N}$ & $\mathrm{I}$ & $\mathrm{P}$ & & \\
\hline $2 / 06-07$ & & G & & Q & $\mathrm{K}$ & & & $\mathrm{T}$ & & G & $\mathrm{N}$ & $\mathrm{T}$ & $\mathrm{H}$ & $S$ & $\mathrm{~F}$ & E & & $\mathrm{H}$ & G & & $\mathrm{N}$ & $\mathrm{F}$ & & I & $\mathrm{R}$ & $\mathrm{N}$ & I & $\mathrm{P}$ & & I \\
\hline 65/08-09 & $\mathrm{X}$ & E & & Q & $\mathrm{N}$ & & & $\mathrm{T}$ & I & & $\mathrm{N}$ & $\mathrm{T}$ & $\mathrm{H}$ & $S$ & $\mathrm{~F}$ & $\mathrm{~N}$ & & $\mathrm{H}$ & G & & $\mathrm{N}$ & $\mathrm{F}$ & & I & $\mathrm{R}$ & $\mathrm{N}$ & I & $\mathrm{P}$ & & \\
\hline $72 / 08-09$ & $\mathrm{X}$ & E & & Q & $\mathrm{K}$ & & & $\mathrm{T}$ & I & & & $\mathrm{T}$ & $\mathrm{H}$ & & $\mathrm{F}$ & Q & & $\mathrm{H}$ & G & & & $\mathrm{F}$ & & I & $\mathrm{R}$ & $\mathrm{N}$ & I & $\mathrm{P}$ & Q & \\
\hline 73/08-09 & $\mathrm{X}$ & $\mathrm{E}$ & & Q & $\mathrm{K}$ & & & $\mathrm{T}$ & I & & $\mathrm{N}$ & $\mathrm{T}$ & $\mathrm{H}$ & & $\mathrm{F}$ & Q & & $\mathrm{H}$ & G & & $\mathrm{N}$ & $\mathrm{F}$ & & I & $\mathrm{R}$ & $\mathrm{N}$ & I & $\mathrm{P}$ & & \\
\hline
\end{tabular}

S31N indicates resistance to adamantanes.

\section{Discussion}

Surveillance data on amantadine resistance mutations in the M2 gene of influenza A H3N2 viruses in the present study showed clearly that the spread and predominance of amantadine-resistant viruses in Dublin, Ireland, emerged in the 2005/2006 season with $73 \%$ resistance, similar to data reported from Europe [15] and worldwide $[4,16]$ in the same influenza season. Interestingly, the first isolate of influenza A H3N2 resistant to amantadine in this study, 63/03-04, showed the potential for rapid development of resistance to adamantanes, particularly in immunocompromised hosts. It has been shown that viruses cultivated in MDCK cells in the presence of amantadine developed variants in the amino acids of the transmembrane domain of the M2 protein where mutations arose at positions 27 (V27A) and 31 (S31N) [17]. In the present study, the immunocompromised patient received only one dose of amantadine, yet a resistant subpopulation of virus was still detected almost 3 weeks later. The initial isolate was amantadine-sensitive (S31S). This showed the stability of influenza A carrying the
S31N mutation and the potential for transmission from asymptomatic hosts shedding the virus [17]. It was also consistent with the rapid development of adamantane resistance, particularly in immuncompromised hosts, reported previously [18]. Phylogenetic analysis of the HA gene of sample 63/03-04 confirmed its similarity with the A/Fujian/411/2002 strain, a clade $S$ virus, typically amantadine-sensitive as shown in this study. The amino acids serine and aspartic acid at positions 193 and 225 in the HA1 region, respectively, classified the isolate in clade S. However, substitutions at these residues, S193F and D225N, are believed to confer a selective advantage for the spread of strains carrying the S31N mutation [10]. Therefore, amantadine resistance in isolates belonging to clade $S$ was only seen when patients were treated with the antiviral and acquired resistance.

Dual resistance mutations to amantadine, V27A and S31N, were identified in isolate 14/06-07 of subtype $\mathrm{H} 3 \mathrm{~N} 2$, but were not of any clinical consequence. The V27A mutation is known to be the most frequent M2 mutation conferring amantadine resistance in seasonal $\mathrm{H} 1 \mathrm{~N} 1$ isolates, whereas the $\mathrm{S} 31 \mathrm{~N}$ mutation predominates 


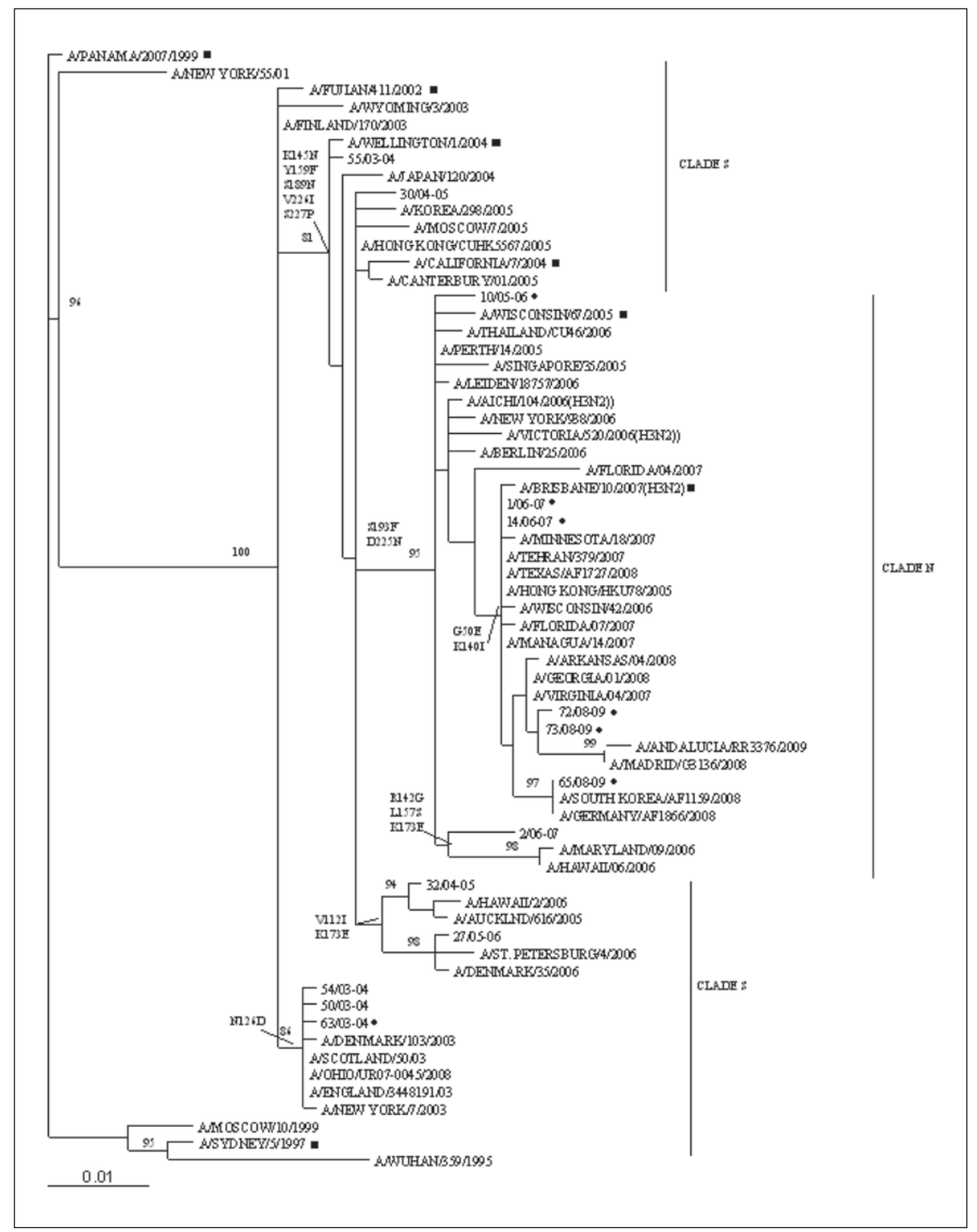

Fig. 2. A maximum likelihood tree based on the partial HA gene ( $989 \mathrm{bp}$ ) of 14 influenza A H3N2 isolates from St. James's Hospital between 2003/2004 and 2008/2009 influenza seasons using A/Panama/2007/1999 (GenBank EF626612) vaccine strain as an outgroup. Bootstrap resampling was carried out using 1,000 replicates of the dataset and the values are written as a percentile beside the appropriate branch. Bootstrap values $<70 \%$ are not shown. Several representative influenza A H3N2 viruses were included. $\bullet=$ Amantadine-resistant viruses, possessing the S31N M2 gene mutation; $\mathbf{\square}=$ vaccine strains used in the tree. Scale bar indicates 0.01 nucleotide substitutions per site. 
in the H3N2 subtype [19]. The finding in this study, albeit based on one patient infected with subtype H3N2, contrasts with findings in animal models using influenza A H1N1 viruses. One study investigated the effects of common amantadine resistance M2 mutations on the phenotypic level of drug resistance [20]. They used seven recombinant $\mathrm{H} 1 \mathrm{~N} 1$ viruses, including the wild type, five single and one double (V27A/S31N) mutants. All mutants exhibited high-level resistance to amantadine, with more than 100 -fold reductions in susceptibility compared to the results with wild-type strain [20]. However, the levels of resistance of the double V27A/S31N mutant were lower than that of each of the corresponding single mutants by factors of 10- and 2-fold, respectively. Therefore, the combination of these two M2 mutations did not confer a synergistic effect in terms of resistance.

The I51V substitution identified in the majority of influenza A H3N2 isolates containing S31N in this study has been reported previously as a marker of change from clade $\mathrm{S}$ (amantadine-sensitive) to clade $\mathrm{N}$ (amantadineresistant) of human influenza $\mathrm{H} 3 \mathrm{~N} 2$ viruses that co-circulated worldwide between 2000 and 2005 [21]. The I51V substitution maps to an HLA-A11-restricted cytotoxic T cell epitope [21]. Therefore, substitutions in this amino acid may be markers of influenza A cytotoxic T cell escape mutants that rapidly become fixed in human populations, by prolonging their infection and infectious period in the human host [22]. Therefore, the I51V M2 substitution may be independently enhancing the persistence and spread of the amantadine-resistant S31N viruses. In the present study, the I51V substitution was not found in any influenza A H3N2-positive samples until the emergence of primary adamantane resistance when it co-existed with S31N. This finding contrasts with a previous study where it was found that the I51V mutation emerged in 2000 before the emergence of the S31N mutation in 2003 [9]. Thereafter, both substitutions co-existed in virtually all Hong Kong isolates up to the end of 2006. The earlier emergence of the I51V mutation may have been necessary to allow the subsequent emergence of the S31N mutation. The I51V substitution was more common than S31N, where it always either occurred alone or with S31N, but S31N never appeared without I51V [9].

Most sensitive viruses (54/03-04, 50/03-04, 55/03-04, $32 / 04-05,27 / 04-05$, and 30/04-05) clustered into clade S. Surprisingly, one virus in the 2003/2004 season (63/0304 ) also clustered into clade $S$ but contained the resistance mutation S31N. It was a sample collected from a patient who had acquired resistance after treatment with amantadine. In addition, isolate $2 / 06-07$ was particularly in-

Genetic Analysis of

Amantadine-Resistant Influenza A teresting because it may have arisen as a result of a reassortment event. In this study, 2/06-07 was grouped into clade $\mathrm{N}$ based on the presence of S193F and D225N in the HAl gene, but did not have genotypic evidence of amantadine resistance. The inclusion of amantadine-sensitive strains in the $\mathrm{N}$ lineage was unusual as previous studies had demonstrated that all viruses in clade $\mathrm{N}$ were amantadine resistant $[10,23]$. Therefore, the 14 strains of influenza A H3N2 were classified into 4 groups in this study: clade N resistant with both S193F and D225N mutations in the HA1 and S31N amantadine-resistant mutation (group A); clade N sensitive with HA1 mutations S193F and D225N and no amantadine-resistant mutation in the M2 gene (group B); clade S-sensitive strains with wildtype amino acids in the HA1 and M2 genes (group C), and clade S-resistant strains with S31N mutation in the $\mathrm{M} 2$ gene (group D). Groups B and D are surprising findings since the $\mathrm{N}$ lineage influenza $\mathrm{A}(\mathrm{H} 3 \mathrm{~N} 2)$ viruses have been associated mainly with amantadine resistance and $S$ lineage strains are typically sensitive to amantadine. It is possible that the events in group B may have arisen from reassortment events, whereas the discrepancy in group D is likely to be a result of acquired resistance. A similar finding was made in a recent study in Japan [24] carried out on isolates collected from the 2005/2006 and $2006 / 2007$ seasons with the aim of further clarifying whether this double amino acid substitution (S193F and $\mathrm{D} 225 \mathrm{~N}$ ) in the HA1 portion of the HA gene had a role in maintaining the S31N mutation in M2. Interestingly, the Japanese study divided their samples into three groups, including amantadine-sensitive isolates of clade $\mathrm{N}$ lineage, similar to what was observed in the present study. In the Japanese study, the genomic events that may have resulted in the $\mathrm{N}$ (Sens) (amantadine-sensitive) group of samples was examined by amplifying and sequencing the full lengths of all 10 genes of some randomly selected samples [24]. It was found that the M2 gene coding for amantadine resistance was replaced by a reassortment event with a gene that did not code for resistance. This event resulted in the $\mathrm{N}$ (Sens) (amantadine-sensitive) strains with the HA1 S193F and D225N mutations without the M2 S31N mutation which may also have occurred in isolate 2/06-07 in the present study but requires full genome sequencing to prove.

However, this link between amantadine resistance and the evolution of influenza A H3N2 isolates could not be explored fully in the present study as it only included two positive H3N2 samples from the 2004/2005 season. This made it difficult to observe the phylogenetic event that occurred during this season. During this study, the 
$2004 / 2005$ season fell between a season where very little amantadine resistance was observed (2003/2004) and a season where $83 \%$ amantadine resistance was observed. Therefore, the 2004/2005 season may have been important in demonstrating possible reassortment events that occurred and led to the spread of amantadine resistance throughout the influenza A H3N2 population as shown in a recent study [25].

Comparison of the nucleotide and amino acid sequences of the HA1 region of the HA and NA genes of isolates in this study (NA data not shown) and other strains circulating in the same seasons, including vaccine strains, showed that Irish isolates were all closely related to the vaccine strains. However, phylogenetic analysis of both HA and NA genes showed some degree of 'antigenic drift' from season to season. Variations in the HA of the influenza A H3N2 isolates examined in this study were located predominantly in or near antigenic sites of the glycoprotein. These amino acid changes may affect the antigenicity or transmissibility of the virus [26]. It was observed that all isolates in this study had substitutions in 11 amino acid residues when compared with the A/ Panama/2007/1999 vaccine strain. All of these residues were also either at or near the important antigenic sites of the HA surface glycoprotein. These substitutions indicate that the viruses circulating since the 2003/2004 influenza season in this study have evolved since the development of the 2003/2004 vaccine strain A/Panama/2007/1999.

In summary, the present study shows how resistance to a class of antiviral agents among influenza viruses can appear without selective pressure from prior use of these drugs and how these resistant strains can disseminate rapidly in the human population. This underlines the need for continuous surveillance of susceptibility to all antiviral agents used for treatment of influenza virus infections. Moreover, this study showed conserved sequences combined with variations due to amino acid substitutions in or proximal to antigenic sites of the HA gene, but high similarity with vaccine strains available during the study period.

\section{References}

$>1$ Belshe RB, Smith MH, Hall CB, Betts R, Hay AJ: Genetic basis of resistance to rimantadine emerging during treatment of influenza virus infection. J Virol 1988;62:1508-1512.

-2 Gubareva LV, Kaiser L, Hayden FG: Influenza virus neuraminidase inhibitors. Lancet 2000;355:827-835.

3 Van Voris LP, Betts RF, Hayden FG, Christmas WA, Douglas RG Jr: Successful treatment of naturally occurring influenza A/ USSR/77 H1N1. JAMA 1981;245:1128-1131.

4 Bright RA, Shay DK, Shu B, Cox NJ, Klimov AI: Adamantane resistance among influenza A viruses isolated early during the 20052006 influenza season in the United States. JAMA 2006;295:891-894.

$\checkmark 5$ Saito R, Li D, Suzuki Y, Sato I, Masaki H, Nishimura H, Kawashima T, Shirahige Y, Shimomura C, Asoh N, Degawa S, Ishikawa H, Sato M, Shobugawa Y, Suzuki H: High prevalence of amantadine-resistance influenza a $(\mathrm{H} 3 \mathrm{~N} 2)$ in six prefectures, Japan, in the 2005-2006 season. J Med Virol 2007;79: 1569-1576.

6 Deyde VM, Xu X, Bright RA, Shaw M, Smith CB, Zhang Y, Shu Y, Gubareva LV, Cox NJ, Klimov AI: Surveillance of resistance to adamantanes among influenza $\mathrm{A}(\mathrm{H} 3 \mathrm{~N} 2)$ and $\mathrm{A}(\mathrm{H} 1 \mathrm{~N} 1)$ viruses isolated worldwide. J Infect Dis 2007; 196:249-257.

7 Swofford DL: PAUP*: Phylogenetic Analysis Using Parsimony (* and other methods). Version 40b10. Sunderland, Sinaur Associates Inc., 2002.
$>8$ Posada D, Crandall KA: MODELTEST: testing the model of DNA substitution. Bioinformatics 1998;14:817-818.

$>9$ Tang JW, Ngai KL, Wong JC, Lam WY, Chan PK: Emergence of adamantane-resistant influenza A(H3N2) viruses in Hong Kong between 1997 and 2006. J Med Virol 2008;80: 895-901.

10 Saito R, Li D, Shimomura C, Masaki H, Le MQ, Nguyen HL, Nguyen HT, Phan TV, Nguyen TT, Sato M, Suzuki Y, Suzuki H: An off-seasonal amantadine-resistant H3N2 influenza outbreak in Japan. Tohoku J Exp Med 2006;210:21-27.

11 Bush RM, Bender CA, Subbarao K, Cox NJ, Fitch WM: Predicting the evolution of human influenza A. Science 1999;286:19211925.

$>12$ Wiley DC, Wilson IA, Skehel JJ: Structural identification of the antibody-binding sites of Hong Kong influenza haemagglutinin and their involvement in antigenic variation. Nature 1981;289:373-378.

>13 Li D, Saito R, Le MT, Nguyen HL, Suzuki Y, Shobugawa Y, Dinh DT, Hoang PV, Tran HT, Nghiem HK, Hoang LT, Huynh LP, Nguyen HT, Nishikawa M, Suzuki H: Genetic analysis of influenza $\mathrm{A} / \mathrm{H} 3 \mathrm{~N} 2$ and $\mathrm{A} / \mathrm{H} 1 \mathrm{~N} 1$ viruses circulating in Vietnam from 2001 to 2006. J Clin Microbiol 2008;46:399-405.

14 WHO: WHO Recommended composition of influenza virus vaccines for use in the 20052006 influenza season. Weekly Epidemiol Record 2005;80:71-75.
15 Schmidtke M, Zell R, Bauer K, Krumbholz A, Schrader C, Suess J, Wutzler P: Amantadine resistance among porcine $\mathrm{H} 1 \mathrm{~N} 1, \mathrm{H} 1 \mathrm{~N} 2$, and $\mathrm{H} 3 \mathrm{~N} 2$ influenza $\mathrm{A}$ viruses isolated in Germany between 1981 and 2001. Intervirology 2006;49:286-293.

16 Bright RA, Medina MJ, Xu X, Perez-Oronoz G, Wallis TR, Davis XM, Povinelli L, Cox NJ, Klimov AI: Incidence of adamantane resistance among influenza A (H3N2) viruses isolated worldwide from 1994 to 2005: a cause for concern. Lancet 2005;366:11751181.

17 Ilyushina NA, Bovin NV, Webster RG, Govorkova EA: Combination chemotherapy, a potential strategy for reducing the emergence of drug-resistant influenza A variants. Antiviral Res 2006;70:121-131.

18 CDC: High levels of adamantane resistance among influenza A (H3N2) viruses and interim guidelines for use of antiviral agents - United States, 2005-06 influenza season. MMWR Morb Mortal Wkly Rep 2006;55: 44-46.

19 Saito R, Sakai T, Sato I, Sano Y, Oshitani H, Sato M, Suzuki H: Frequency of amantadineresistant influenza A viruses during two seasons featuring cocirculation of $\mathrm{H} 1 \mathrm{~N} 1$ and H3N2. J Clin Microbiol 2003;41:2164-2165.

20 Abed Y, Goyette N, Boivin G: Generation and characterization of recombinant influenza A (H1N1) viruses harboring amantadine resistance mutations. Antimicrob Agents Chemother 2005;49:556-559. 
-21 Holmes EC, Ghedin E, Miller N, Taylor J, Bao Y, St George K, Grenfell BT, Salzberg SL, Fraser CM, Lipman DJ, Taubenberger JK: Whole-genome analysis of human influenza A virus reveals multiple persistent lineages and reassortment among recent $\mathrm{H} 3 \mathrm{~N} 2$ viruses. PLoS Biol 2005;3:e300.

22 Gog JR, Rimmelzwaan GF, Osterhaus AD, Grenfell BT: Population dynamics of rapid fixation in cytotoxic T lymphocyte escape mutants of influenza A. Proc Natl Acad Sci USA 2003;100:11143-11147.
23 Simonsen L, Viboud C, Grenfell BT, Dushoff J, Jennings L, Smit M, Macken C, Hata $M$, Gog J, Miller MA, Holmes EC: The genesis and spread of reassortment human influenza A/H3N2 viruses conferring adamantane resistance. Mol Biol Evol 2007;24:1811-1820.

-24 Furuse Y, Suzuki A, Kamigaki T, Shimizu M, Fuji N, Oshitani H: Reversion of influenza A (H3N2) virus from amantadine resistant to amantadine sensitive by further reassortment in Japan during the 2006-to-2007 influenza season. J Clin Microbiol 2009;47: 841-844.
Jonges $\mathrm{M}$, van der Lubben IM, Dijkstra F, Verhoef L, Koopmans M, Meijer A: Dynamics of antiviral-resistant influenza viruses in the Netherlands, 2005-2008. Antiviral Res 2009;83:290-297.

26 Medeiros R, Naffakh N, Manuguerra JC, van der Werf S: Binding of the hemagglutinin from human or equine influenza $\mathrm{H} 3$ viruses to the receptor is altered by substitutions at residue 193. Arch Virol 2004;149:1663-1671. 